\title{
Probing dense matter in compact star cores with radio pulsar data
}

\author{
Mark Alford and Kai Schwenzer \\ Department of Physics, Washington University, St. Louis, Missouri, 63130, USA
}

\begin{abstract}
Astrophysical observations of compact stars provide, in addition to collider experiments, the other big source of information on matter under extreme conditions. The largest and most precise data set about neutron stars is the timing data of radio pulsars. We show how this unique data can be used to learn about the ultra-dense matter in the compact star interior. The method relies on astro-seismology based on special global oscillation modes (r-modes) that emit gravitational waves. They would prevent pulsars from spinning with their observed high frequencies, unless the damping of these modes, determined by the microscopic properties of matter, can prevent this. We show that for each form of matter there is a distinct region in a frequency/spindown-rate diagram where r-modes can be present. We find that stars containing ungapped quark matter are consistent with both the observed radio and $\mathrm{x}$-ray data, whereas, even when taking into account the considerable uncertainties, neutron star models with standard viscous damping are inconsistent with both data sets and additional damping mechanisms would be required.
\end{abstract}

Keywords: Dense matter, Compact stars, Pulsars

\section{Introduction}

Radio observations led to the discovery of pulsars nearly fifty years ago [1]. At the time the only explanation were neutron stars [2], but since the formulation of QCD the study of strongly interacting matter under extreme conditions has become a very active research area. Whereas heavy ion experiments mainly probe the hot plasma phase, the interior of compact stars would be an ideal laboratory to study cold dense matter and many interesting options for the composition of such ultradense compact objects were proposed [3], including most notably deconfined quark matter. However, a search for novel phases of matter in compact stars requires appropriate astrophysical observables. Since the initial discovery of pulsars a wealth of radio pulsar data has been compiled [4] that is still our best source of information on compact stars and even among the most precise data sets in physics. In particular, millisecond pulsars, that can be billions of years old, are extremely stable systems for which not only the frequency but also the spindown rate is known. To use this timing data to learn about the star's interior composition poses a challenge, since the radio emission originates far away in the pulsar's magnetosphere, whereas no electromagnetic radiation can leave the dense core of a compact star. Based on our recent article [5], we show here how pulsar timing data can nevertheless provide information about the dense matter in the interior and could allow us to discriminate different star compositions.

The method is based on astroseismology, which exploits that mechanical oscillation modes can probe the opaque dense interior of the star. Even though such seismic oscillations cannot be observed, certain global modes emit gravitational waves. R-modes [6] are of particular interest since they are unstable due to the Friedman-Schutz mechanism [7] and grow spontaneously. The gravitational waves emitted by r-mode oscillations may be directly detectable $[8,9]$ in future interferometers [10], but they also carry away angular momentum and would prevent the spin-up of a star by accretion to millisecond frequencies if r-modes are not efficiently damped. Therefore, the fact that we observe millisecond pulsars means that such damping is present and the timing data of pulsars contains information about the 
damping in the interior. This damping stems from microscopic reactions within a given phase, described particularly by its viscosities. It depends therefore not only on the equation of state, but on the low energy degrees of freedom, which can differ drastically for different forms of matter, and this provides an efficient way to discriminate them.

\section{Dynamic versus static r-mode astroseismology}

We will first review the conventional way to perform r-mode seismology [11]. Despite the damping due to viscosities or other dissipative mechanisms, r-modes are generally unstable at sufficiently large frequency in characteristic static instability regions in a $T$ - $f$-diagram. These are shown for a few models of dense matter on the left panel of figure 1. These curves can be compared to data from low mass x-ray binaries (LMXB) [12] which currently are spun up and heated by accretion and therefore emit $\mathrm{x}$-ray radiation that allows to estimate their temperature. The data set is rather small and the temperatures are still uncertain since they require modeling the $\mathrm{x}$-ray spectrum. The dashed curve is the instability boundary for a neutron star with standard viscous damping. Most sources are clearly inside of this boundary and r-modes are therefore unstable at small r-mode amplitude $\alpha$. In this case the r-mode grows exponentially until it is stopped by an amplitude-dependent increase of the damping that saturates the amplitude at a finite value $\alpha_{\text {sat }}[13,14]$. The only way to make this model compatible with the data would be that the corresponding saturation amplitude is so small that it hardly affects the spin evolution (the tiny $r$-mode scenario). This would require a very low value of the order $\alpha_{\text {sat }} \lesssim 10^{-8}-10^{-7}[5,15]$. However, currently proposed mechanisms for the saturation of r-modes can at most saturate r-modes at $\alpha_{\text {sat }} \gtrsim 10^{-6}$ [14] so that the data cannot be explained by standard viscous damping in hadronic matter. Since neutron stars are structurally and compositionally complicated, and involve e.g. a crystalline crust, additional enhanced damping is possible in hadronic matter. Yet, in contrast to viscous damping often even the order of magnitude of these contributions is still uncertain and it will require further study to see if they are relevant. An example is the rubbing of the neutron fluid at the solid crust [16], but as seen by the dotted curve, even under favorable assumptions [5] this mechanism cannot damp the instability in the fastest sources. In contrast many novel forms of matter feature enhanced viscous damping leading to a significantly reduced instability region (the no r-mode scenario), as shown by the solid curve for a model of ungapped interacting quark matter [17].

To probe dense matter in compact star cores with the distinct radio pulsar timing data set requires us to understand the spin evolution of millisecond pulsars in the presence of r-modes after the accretion stopped. The evolution is obtained from global conservation constraints [13] and includes the pulsar spin-frequency $f$, the dimensionless rmode amplitude $\alpha$ [6], and, since the damping of r-modes heats the star, also of the temperature $T$ of the star [13]. The relevant observables such as the moment of inertia, the dissipative damping or the radiative cooling of the star depend on these evolution parameters via simple power laws. Therefore, an "effective theory" approach $[8,5]$ can be employed in order to describe the evolution of a pulsar, which exploits that pulsars are effectively point sources and the complete information about the interior of the star can be encoded in a few ("low energy") constants, that are integrals over the entire star. Using this formalism one can derive general analytic expressions for the r-mode evolution [8, 5]. This is possible since the three coupled evolution parameters $f, \alpha$ and $T$ evolve on very different time scales. As discussed, if the r-mode is unstable, its amplitude saturates quickly at a value $\alpha_{\text {sat }}(f, T)$. It has been shown that for an arbitrary star composition the thermal evolution is always faster than the spin evolution [8]. Therefore, once the accretion stops, the star rapidly cools until it either leaves the instability region or until it reaches the thermal steady state curve, where heating is balanced by radiative cooling, along which it then very slowly spins down. The steady state depends on the r-mode saturation amplitude since the dissipative heating is large for a high amplitude mode. Following the evolution along this spindown curve until it leaves the static instability region allows to determine the $\mathrm{r}$ mode spindown rate $\dot{f}_{\mathrm{R}}$ at this point and correspondingly to compute an analogous dynamic instability region in a $\dot{f}_{\mathrm{R}}-f$ diagram. The striking feature of the dynamic boundary curves is that-like the static versions-they are completely independent of the saturation mechanism and the saturation amplitude [5].

Dynamic instability regions for the star compositions discussed previously are shown on the right panel of fig. 1 . As can be seen these curves are qualitatively similar to the corresponding static instability boundaries on the left panel. These curves can now be compared to the comprehensive timing data of millisecond radio pulsars [4], which had in the past been spun up in a LMXB system. It is important to stress that all data points are only upper limits since other spindown mechanisms should be present in these sources as well. For the standard neutron star model (dashed) these upper bounds are again clearly inside the instability region. As discussed in [5], the remarkable aspect of our method is that the mere upper spindown rate limits are sufficient to constrain the interior composition. The reason for this is 

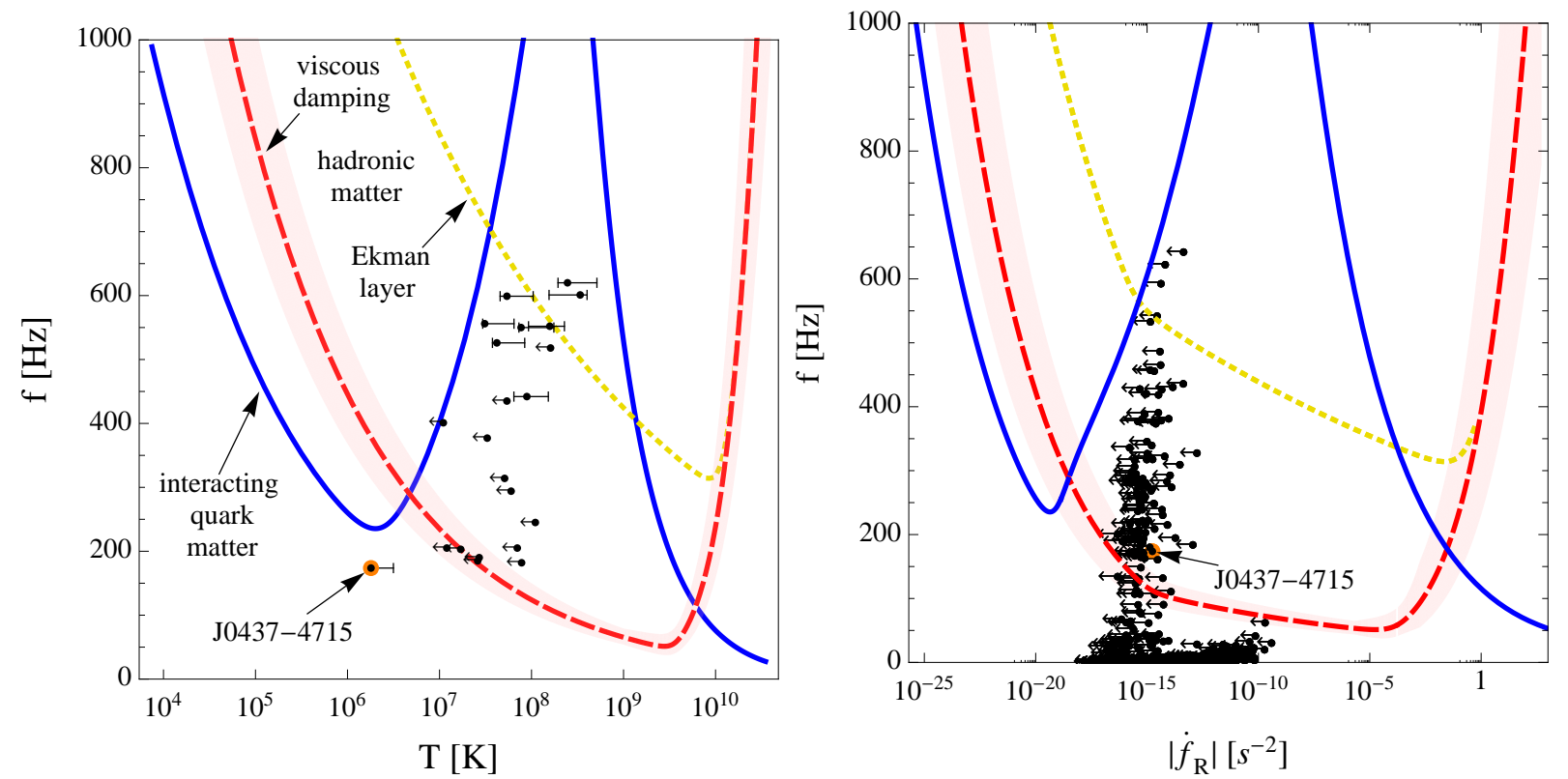

Figure 1. Boundaries of the r-mode instability regions for different star compositions compared to pulsar data. Left: Standard static instability boundary compared to X-ray data [12] with error estimates from different envelope models. Right: Dynamic instability boundary in timing parameter space compared to radio data [4] (all data points are upper limits for the r-mode component of the spindown). The curves represent: $1.4 M_{\odot}$ neutron star (NS) with standard viscous damping [18, 19] (dashed) and with additional boundary layer rubbing [16] at a rigid crust (dotted) as well as $1.4 M_{\odot}$ strange star (SS) with long-ranged NFL interactions causing enhanced damping [20, 17] (using $\alpha_{s}=1$ ) (solid). More massive stars are not qualitatively different, as is seen by the shaded band which shows for the standard neutron star an estimate of all uncertainties in both the micro- and macroscopic description. The encircled points denote the only millisecond radio pulsar J0437-4715 with a temperature estimate.

that millisecond pulsars are spun up at rather high temperature, as seen on the left panel of fig. 1, and cannot cool out of the static instability region for all relevant saturation amplitudes $\alpha_{\text {sat }} \gtrsim 10^{-10}$ [5]. This value is many orders of magnitude smaller than what known saturation mechanisms can provide [14]. Consequently fast sources that happen to lie in the dynamic instability region must also lie inside of the static region. If, in contrast, a source is outside of the dynamic instability region for a given star composition, r-modes cannot be the dominant spindown mechanism and such an instability region is therefore consistent with the data, as is the case for ungapped quark matter [17] (solid).

In order to clearly discriminate different forms of dense matter requires control over the significant uncertainties in both our understanding of strongly interacting dense matter and of the properties of compact stars. A very favorable feature of the semi-analytic results for the instability regions [5] is that they can be extremely insensitive [21, 8] to the tremendous uncertainties in the underlying microscopic and macroscopic parameters (e.g. the bulk viscosity of dense matter involves strong interaction corrections and allows only an order of magnitude estimate). This insensitivity is illustrated for the instability regions of the minimal neutron star model in fig. 1, where the uncertainty range is shown by the shaded bands and we use the uncertainty ranges for the underlying parameters estimated in [8]. These bands show that the minimal hadronic reference model is indeed inconsistent with the data. The uncertainties in the quark matter model are significantly bigger [17], but over large parts of the parameter space they are consistent with the data, so that within uncertainties ungapped quark matter would be a viable candidate for the interior of compact stars.

Another important requirement to use r-mode seismology to detect the enhanced damping of exotic forms of matter, is that we can rule out the tiny r-mode scenario, i.e. ensure r-modes are not saturated at such low amplitudes that they would be virtually irrelevant. For this it is interesting that even very low amplitude r-modes, that would not affect the spindown evolution, would significantly heat a source and the corresponding temperature is directly given in terms of $f$ and $\dot{f}_{\mathrm{R}}$ and independent of the r-mode saturation mechanism [5]. The corresponding upper bounds from the timing data lead to temperatures that are considerably larger than standard cooling models predict and which are large enough that it could be possible to detect the thermal x-ray radiation [5]. Therefore, either the detection or strict upper limits on the thermal $\mathrm{x}$-ray observation of nearby isolated radio pulsars should tell us if $\mathrm{r}$-modes can be present. 


\section{Conclusions}

We have discussed how the comprehensive and precise timing data of radio pulsars can be used to probe the dense matter in the interior of compact stars. We show that standard neutron matter with mere viscous damping is inconsistent with the data and enhanced damping is required. As demonstrated, interacting ungapped quark matter is consistent with the data, but there are many other options, like in particular enhanced damping mechanisms in hadronic matter e.g. due to hyperons [22], superfluidity, the neutron star crust [16] or large magnetic fields [23]. We will have to strongly improve our understanding of the damping in compact stars by detailed future studies, but with the combination of thermal x-ray, radio timing and future gravitational wave data we have an excellent data base, so that a clear discrimination of different forms of matter becomes a viable possibility.

\section{Acknowledgements}

This material is based upon work supported by the U.S. Department of Energy, Office of Science, Office of Nuclear Physics and High Energy Physics under Award Number \#DE-FG02-91ER40628 and \#DE-FG02-05ER41375.

\section{References}

[1] A. Hewish, S. Bell, J. Pilkington, P. Scott, R. Collins, Observation of a rapidly pulsating radio source, Nature 217 (1968) $709-713$. doi:10.1038/217709a0.

[2] J. Lattimer, M. Prakash, The physics of neutron stars, Science 304 (2004) 536-542. arXiv:astro-ph/0405262, doi:10.1126/science.1090720.

[3] M. G. Alford, A. Schmitt, K. Rajagopal, T. Schafer, Color superconductivity in dense quark matter, Rev. Mod. Phys. 80 (2008) $1455-1515$. arXiv:0709.4635, doi:10.1103/RevModPhys.80.1455.

[4] R. N. Manchester, G. B. Hobbs, A. Teoh, M. Hobbs, The ATNF Pulsar Catalogue, Astron. J. 129 (2005) 1993. arXiv:astro-ph/0412641, doi: $10.1086 / 428488$.

[5] M. G. Alford, K. Schwenzer, What flashes of pulsars can teach us about their interiorarXiv:1310.3524.

[6] N. Andersson, A new class of unstable modes of rotating relativistic stars, Astrophys. J. 502 (1998) 708-713. arXiv:gr-qc/9706075, doi:10.1086/305919.

[7] J. L. Friedman, B. F. Schutz, Secular instability of rotating Newtonian stars, Astrophys. J. 222 (1978) 281. doi:10.1086/156143.

[8] M. G. Alford, K. Schwenzer, Gravitational wave emission and spindown of young pulsars, Astrophys.J. 781 (2014) 26. arXiv:1210.6091, doi:10.1088/0004-637X/781/1/26.

[9] M. G. Alford, K. Schwenzer, Gravitational wave emission from oscillating millisecond pulsarsarXiv:1403.7500.

[10] G. M. Harry, Advanced LIGO: The next generation of gravitational wave detectors, Class.Quant.Grav. 27 (2010) 084006. doi:10.1088/02649381/27/8/084006.

[11] N. Andersson, K. D. Kokkotas, The R-mode instability in rotating neutron stars, Int. J. Mod. Phys. D10 (2001) 381-442. arXiv:gr-qc/0010102, doi:10.1142/S0218271801001062.

[12] B. Haskell, N. Degenaar, W. C. G. Ho, Constraining the physics of the r-mode instability in neutron stars with X-ray and ultraviolet observations, Mon. Not. Roy. Astron. Soc. 424 (2012) 93-103. arXiv:1201.2101, doi:10.1111/j.1365-2966.2012.21171.x.

[13] B. J. Owen, et al., Gravitational waves from hot young rapidly rotating neutron stars, Phys. Rev. D58 (1998) 084020. arXiv:gr-qc/9804044, doi:10.1103/PhysRevD.58.084020.

[14] R. Bondarescu, I. Wasserman, Nonlinear Development of the R-Mode Instability and the Maximum Rotation Rate of Neutron Stars, Astrophys.J. 778 (2013) 9. arXiv:1305.2335, doi:10.1088/0004-637X/778/1/9.

[15] S. Mahmoodifar, T. Strohmayer, Upper Bounds on $r$-mode Amplitudes from Observations of Low-mass X-Ray Binary Neutron Stars, Astrophys.J. 773 (2013) 140. arXiv:1302.1204, doi:10.1088/0004-637X/773/2/140.

[16] L. Lindblom, B. J. Owen, G. Ushomirsky, Effect of a neutron star crust on the r mode instability, Phys.Rev. D62 (2000) 084030. arXiv:astro$\mathrm{ph} / 0006242$, doi:10.1103/PhysRevD.62.084030.

[17] K. Schwenzer, How long-range interactions tune the damping in compact starsarXiv:1212.5242.

[18] P. S. Shternin, D. G. Yakovlev, Shear viscosity in neutron star cores, Phys. Rev. D78 (2008) $063006 . \quad$ arXiv:0808.2018, doi:10.1103/PhysRevD.78.063006.

[19] R. F. Sawyer, Bulk viscosity of hot neutron-star matter and the maximum rotation rates of neutron stars, Phys. Rev. D39 (1989) $3804-3806$. doi:10.1103/PhysRevD.39.3804.

[20] H. Heiselberg, C. J. Pethick, Transport and relaxation in degenerate quark plasmas, Phys. Rev. D48 (1993) $2916-2928$. doi:10.1103/PhysRevD.48.2916.

[21] M. Alford, S. Mahmoodifar, K. Schwenzer, Viscous damping of r-modes: Small amplitude instability, Phys.Rev. D85 (2012) 024007. arXiv:1012.4883, doi:10.1103/PhysRevD.85.024007.

[22] L. Lindblom, B. J. Owen, Effect of hyperon bulk viscosity on neutron-star r- modes, Phys. Rev. D65 (2002) 063006. arXiv:astro-ph/0110558, doi:10.1103/PhysRevD.65.063006.

[23] L. Rezzolla, F. K. Lamb, S. L. Shapiro, R mode oscillations in rotating magnetic neutron stars, Astrophys.J. 531 (2000) L141-144. arXiv:astro-ph/9911188, doi:10.1086/312539. 